\title{
Therapeutic Antibodies and Cancer Vaccines
}

\author{
Mahnoor Patel
}

Department of Biotechnology, Veer Narmad South Gujarat University, Surat 395007, Gujarat, India.

Email: patelmahnoor786@gmail.com

\section{Abstract}

Antibodies are proteins synthesized in blood against specific antigens. Antigen binding causes activation, proliferation and differentiation of the B cells. After activation, $\mathrm{B}$ cells becomes plasma cells and secrete antibodies. Therapeutic antibodies are monoclonal antibodies and use as passive immunotherapy for the treatment of cancer. It is well established option in clinical oncology. Anticancer vaccines are less advanced because of some exception of successfully applied prophylactic vaccines against oncogenic virus infections. The formation of therapeutic vaccine is still a great challenge due to the self nature of tumor antigens. Therapeutic antibodies as vaccines against cancer are produced in healthy cells by using cancer antigens and use for the patient to treat existing cancer by strengthening the natural defense of the body against cancer.
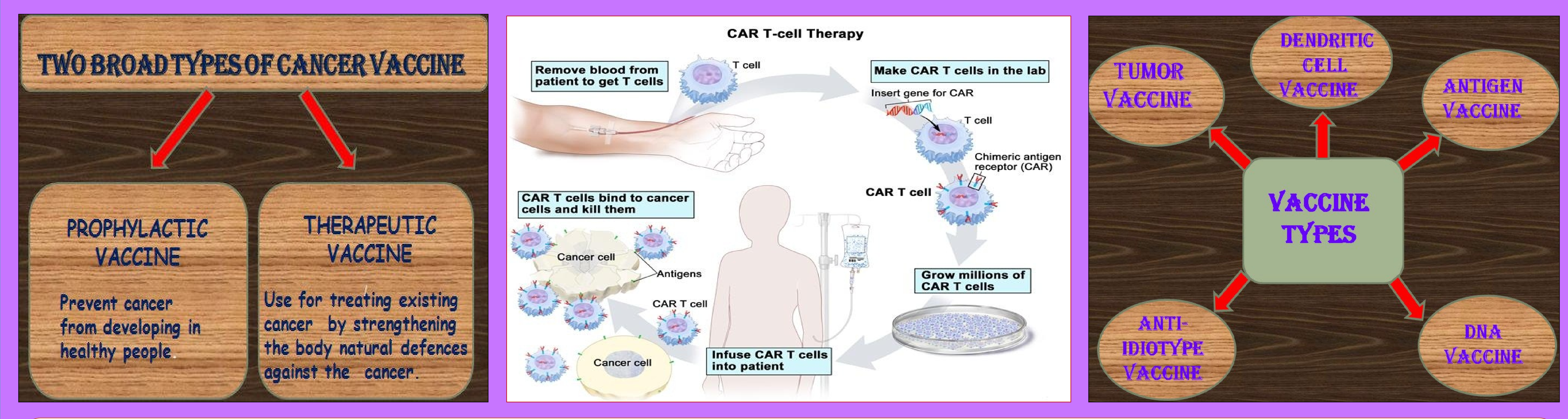

\section{Different Types of Vaccines}

1. Tumor Cell Vaccine: A tumor antigen vaccine is a vaccine made of cancer cells, parts of cancer cells, or modified tumor antigen. This vaccine is circulating tumor antigen vaccine.

2. Antigen Vaccine: Antigen vaccine do not involve the insertion of modified tumor cells. Isolation of specific antigenic gene or a peptide sequence which directly use as a vaccine for benign tumors only.

3. DNA Vaccine: This vaccine are still in experimental stage. Like other vaccines, genes for the desired antigens are located and cloned.

4. Anti-Idiotype Vaccine: The vaccine in which an antibody that binds to the antigen-combining site of another antibody either suppressing or enhancing the immune response. Idiotype are present in variable region of an antibody. Antibody from cancer patient isolated because as person immune system is weakened, it is not able to produce higher concentration of an antibody. As person is suffering from cancer, definitely the immune system activated against tumor antigens.

5. Therapeutic Vaccines: When someone is suffer from cancer then they get therapeutic vaccine to treat that cancer. Therapeutic vaccine is used to treat previously existing cancer. This type of vaccine treat cancer by strengthening the body's natural defense system. Successful vaccines available for breast cancer and cervical cancer.

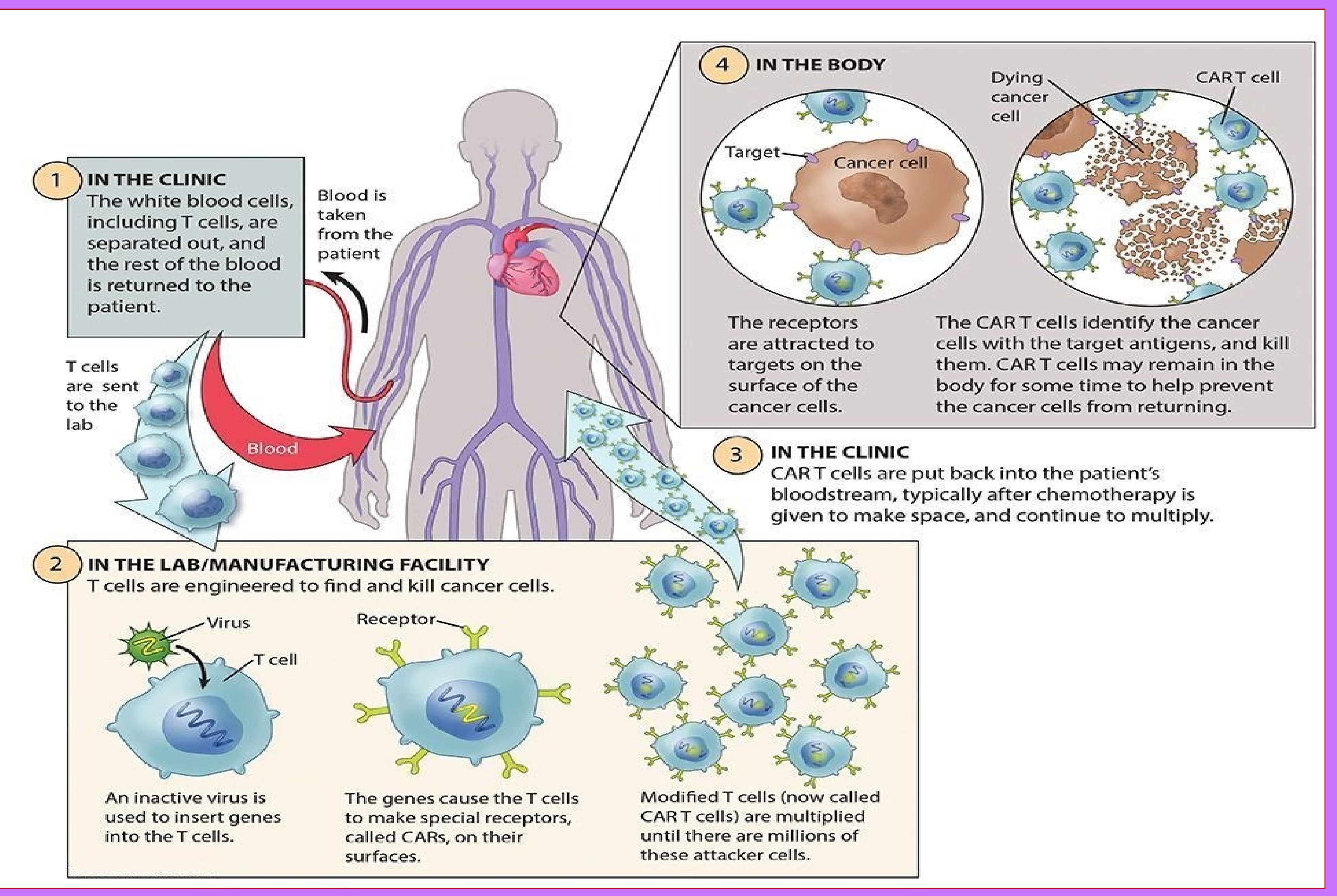

\section{Advantages of Different Types of Vaccines}

1. Whole Tumor Cells: Complete antigen pool of an individual tumor. Activation of a polyclonal and more effective immune response. The immune system rather than the vaccinologist select the most immunogenic tumor-specific antigens.

2. Dendritic Cells: Stimulation of both naïve and memory T cells. Presentation of the vaccine antigens to other cell types of the immune system.

. DNA: Easy and cheap to produce and purify. Require no special handling or storage conditions. Elicitation of both $\mathrm{CD}^{+}$and $\mathrm{CD}^{+}{ }^{+}$immune responses as well as humoral responses.

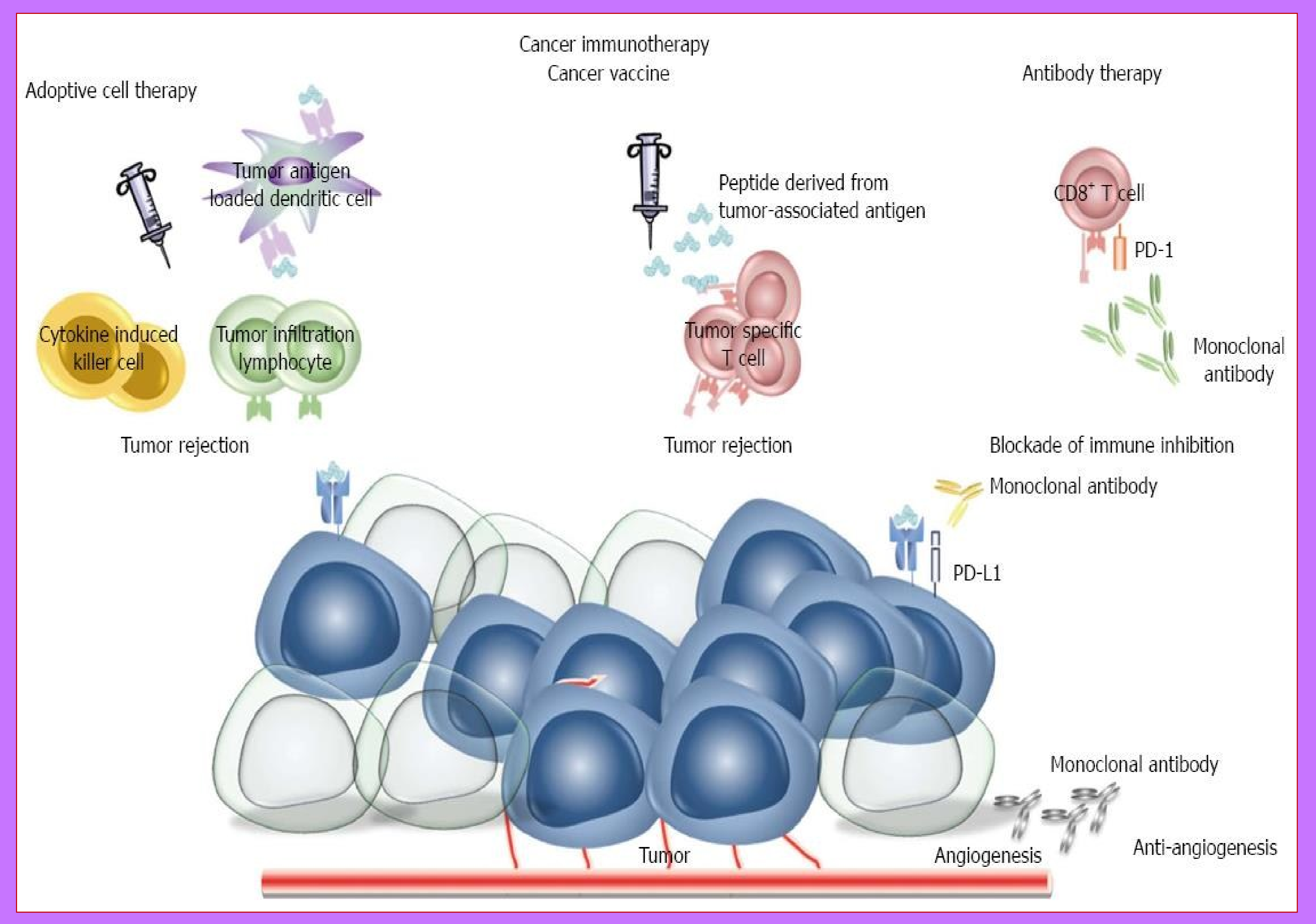

Disadvantages of Different types of Vaccines

1. Whole Tumor Cells: Induction of auto-immunity in the presence of adjuvants. Immune response difficult to monitor. Must be made individually for each patient. Lack of co-stimulatory molecules on solid tumor cells.

2. Dendritic Cells: Must be made individually for each patient. Generation of DC is technically challenging. Money and time consuming treatment.

3. DNA: DNA integration into the cells genome potentially promoting malignancy. Less effective than peptide vaccine at inducing the $\mathrm{CD}^{+} \mathrm{T}$ cell response.

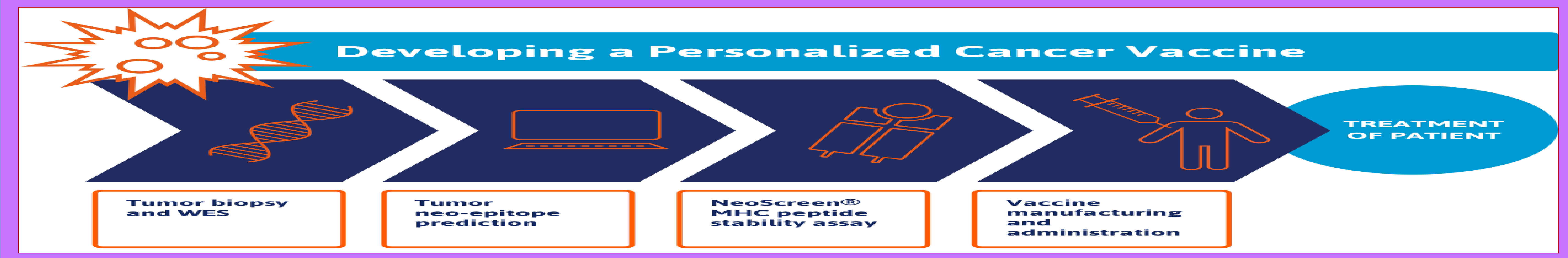

